\title{
HARNACK'S INEQUALITY FOR DEGENERATE SCHRÖDINGER OPERATORS
}

\author{
CRISTIAN E. GUTIERREZ
}

\begin{abstract}
We prove a Harnack inequality for nonnegative weak solutions of certain Schrödinger equations of the form $L u-V u=0$ where $L$ is a second order degenerate elliptic operator in divergence form and $V$ is a potential in certain class.
\end{abstract}

\section{INTRODUCTION}

The purpose of this paper is to establish a Harnack inequality for nonnegative weak solutions of certain degenerate Schrödinger equations of the form

$$
L u-V u=\sum_{i, j=1}^{n} D_{x_{i}}\left(a_{i j}(x) D_{x_{j}} u\right)-V u=0,
$$

$x \in R^{n}$. The coefficients $a_{i j}$ are measurable real-valued functions, the coefficient matrix $a=\left(a_{i j}\right)$ is symmetric and

$$
\lambda^{-1} w(x)|\xi|^{2} \leq\langle a(x) \xi, \xi\rangle \leq \lambda w(x)|\xi|^{2},
$$

where $\lambda>0, \xi=\left(\xi_{1}, \ldots, \xi_{n}\right),\langle$,$\rangle is the Euclidean inner product and w$ is a weight satisfying either

(i) $w \in A_{2}$, that is

$$
\sup _{B}\left(f_{B} w(x) d x\right)\left(f_{B} w(x)^{-1} d x\right)=c_{0}<\infty,
$$

where the supremum is taken over all balls $B$ in $R^{n}$ and $f_{B} w(x) d x$ denotes the average of $w$ over $B$. The constant $c_{0}$ is referred to as the $A_{2}$ constant of $w$; or

(ii) $w(x)=\left|f^{\prime}(x)\right|^{1-2 / n}$, where $f: R^{n} \rightarrow R^{n}$ is a quasiconformal mapping and $\left|f^{\prime}(x)\right|$ denotes the absolute value of the Jacobian determinant of $f$. Quasiconformal means $f=\left(f_{1}, \ldots, f_{n}\right)$ is one-to-one, the distributional derivatives of $f_{i}$ belong to $L_{\mathrm{loc}}^{n}\left(R^{n}\right)$ and there is a constant $C_{0}>0$, called the

Received by the editors November 20, 1987.

1980 Mathematics Subject Classification (1985 Revision). Primary 35B45, 35J70; 35J10; Secondary 42B99, 46E35. 
dilation constant of $f$, such that a.e.

$$
\left[\sum_{i, j=1}^{n}\left(D_{x_{j}} f_{i}\right)^{2}\right]^{1 / 2} \leq C_{0}\left|f^{\prime}(x)\right|^{1 / n}
$$

We shall assume that the potential $V$ satisfies the following condition

$$
\lim _{\delta \rightarrow 0} \sup _{B_{R / 4}\left(x_{0}\right)} \int_{|x-y|<\delta}|V(y)| \int_{|x-y|}^{R} \frac{s^{2}}{w\left(B_{s}(x)\right)} \frac{d s}{s} d y=0,
$$

for every $x_{0} \in R^{n}$ and $R>0, B_{s}(x)$ denotes the ball centered at $x$ with radius $s$.

Let $\Omega$ be an open, bounded and connected set in $R^{n}$. We say that the function $u$ is a weak solution of $L u-V u=0$ in $\Omega$ if $u \in H_{\text {loc }}^{1}(\Omega, w)$ and

$$
-\int\langle a(x) \nabla u(x), \nabla \psi(x)\rangle d x=\int V(x) u(x) \psi(x) d x
$$

for every $\psi \in H_{0}^{1}(\Omega, w)$ (see definitions in $\S 2$ ).

Given $\Omega$ an open bounded subset of $R^{n}$ let $B_{R}$ be the smallest ball containing $\Omega$. If $\eta$ is a nondecreasing function defined for $r>0$ and such that $\lim _{r \rightarrow 0} \eta(r)=0$ then we set

$$
K_{\eta}=\left\{V: \sup _{x \in B_{R}} \int_{|x-y| \leq r}|V(y)| \int_{|x-y|}^{4 R} \frac{s^{2}}{w\left(B_{s}(x)\right)} \frac{d s}{s} d y \leq \eta(r), r>0\right\} .
$$

By $c_{0}$ we denote either the $A_{2}$ constant of $w$ or the dilation constant of $f$ if $w$ satisfies (ii). The main result is the following:

Theorem. Let $\Omega$ be an open, bounded subset of $R^{n}, w$ is a weight satisfying (i) or (ii) and $V \in K_{\eta}$. Then there exist positive constants $r_{0}$ and $\gamma$ only depending on $\lambda, n, c_{0}, \Omega$ and $\eta$ such that if $u$ is any nonnegative solution of (1.1) in $\Omega$ then for any ball $B_{r}$ with $B_{8 r} \subset \Omega$ and $0<r \leq r_{0}$ we have

$$
\sup _{B_{r / 2}} u \leq \gamma \inf _{B_{r / 2}} u
$$

The theorem in the nondegenerate case, i.e. $w \equiv 1$, was obtained by Chiarenza, Fabes and Garofalo in [1]. In this case (1.2) means that $V$ belongs to the Kato-Stummel class. In the degenerate case (1.2) is suggested by the following approximate formula for the Green's function $G_{L}(x, y)$ for $L$ in $B_{R}\left(x_{0}\right)$ valid when $w$ satisfies (i) or (ii),

$$
G_{L}(x, y) \simeq \int_{|x-y|}^{R} \frac{s^{2}}{w\left(B_{s}(x)\right)} \frac{d s}{s},
$$

for $x, y \in B_{R / 4}\left(x_{0}\right)$ (see [4] for a proof of this formula). It is easy to see that if $V / w \in L_{w}^{p}$ locally for $p>(n / 2) \mu$ then $V$ satisfies (1.2). Here $\mu$ means the doubling order of $w$, i.e. $w \in D_{\mu}$ (see $\S 2$ for definitions). 
The proof of our Theorem is based on the method developed in [1] and [6] which basically consists of estimating powers of the solution $u$. One of the ingredients used in the proof is a weighted interpolation inequality (Lemma (3.3)) having some independent interest.

As in the nondegenerate case our result implies the continuity of solutions.

The paper is organized as follows: in $\S 2$ we state some preliminary definitions and results, in $\S 3$ we show an $L^{\infty}$-estimate for solutions and in $\S 4$ we establish some properties of the Green's function for $L-V$ and the infimum estimate.

At this time it is a pleasure to thank E. B. Fabes for his encouragement and many helpful discussions. The author wants to express also his gratitude to the University of Rochester for their kindness and hospitality during his residency for the academic year of 1986-1987.

\section{Preliminaries}

$\Omega$ denotes a bounded, open and connected subset of $R^{n} . L^{p}(\Omega, w)$ denotes the class of functions $f$ such that

$$
\|f\|_{p, w}^{p}=\int_{\Omega}|f(x)|^{p} w(x) d x<\infty .
$$

$\operatorname{Lip}(\bar{\Omega})$ denotes the class of Lipschitz functions in $\bar{\Omega}$. We say $\psi \in \operatorname{Lip}_{0}(\Omega)$ if $\psi \in \operatorname{Lip}(\bar{\Omega})$ and $\psi$ has compact support contained in $\Omega$. For $\psi \in \operatorname{Lip}(\bar{\Omega})$ we define the norm

$$
\int_{\Omega}|\psi(x)|^{2} w(x) d x+\int_{\Omega}|\nabla \psi(x)|^{2} w(x) d x .
$$

$H^{1}(\Omega, w)$ denotes the closure of $\operatorname{Lip}(\bar{\Omega})$ under the norm (2.1). $H_{0}^{1}(\Omega, w)$ denotes the closure of $\operatorname{Lip}_{0}(\Omega)$ under the norm $(2.1) . H^{-1}(\Omega, w)$ denotes the dual space of $H_{0}^{1}(\Omega, w)$. When $w$ satisfies (i) or (ii) and $u \in H^{1}(\Omega, w)$ the gradient of $u$ is uniquely defined (see [5, §2]). It can be shown (see [4, p. 154]) that

$$
\begin{array}{r}
H^{-1}(\Omega, w)=\left\{f_{0}-\operatorname{div} \vec{f}: \vec{f}=\left(f_{1}, \ldots, f_{n}\right), f_{i} / w \in L^{2}(\Omega, w),\right. \\
\quad i=0,1, \ldots, n\} .
\end{array}
$$

We say $u \in H_{\mathrm{loc}}^{1}(\Omega, w)$ if $u \in H^{1}\left(\Omega^{\prime}, w\right)$ for every $\Omega^{\prime}$ with closure contained in $\Omega$. Let $u \in H^{1}(\Omega, w), E \subset \bar{\Omega}$, then $u \geq 0$ on $E$ in the sense of $H^{1}(\Omega, w)$ if there exists a sequence $u_{n} \in \operatorname{Lip}(\bar{\Omega})$ such that $u_{n}(x) \geq 0$ for $x \in E$ and $u_{n} \rightarrow u$ in $H^{1}(\Omega, w)$. If $w$ satisfies (i) or (ii) then Poincaré's inequality holds, i.e. there exist constants $C$ and $\tau>1$ depending only on $c_{0}$ such that

$$
\left(f_{B}\left|u-u_{B}\right|^{2 \tau} w(x) d x\right)^{1 / 2 \tau} \leq C|B|^{1 / n}\left(f_{B}|\nabla u|^{2} w(x) d x\right)^{1 / 2}
$$

for all $u \in H^{1}(\Omega, w), u_{B}=\frac{1}{W(B)} \int_{B} u w d x$. Also, if $u \in H_{0}^{1}(\Omega, w)$ we have Sobolev's inequality

$$
\left(f_{B}|u|^{2 \tau} w(x) d x\right)^{1 / 2 \tau} \leq C|B|^{1 / n}\left(f_{B}|\nabla u|^{2} w d x\right)^{1 / 2} .
$$


For a proof of (2.2) and (2.3) see [5]. We say that the weight $w$ satisfies a doubling condition of order $\mu$ if there exists a constant $C>0$ such that

$$
w\left(B_{t r}\left(x_{0}\right)\right) \leq C t^{n \mu} w\left(B_{r}\left(x_{0}\right)\right)
$$

for every $x_{0} \in R^{n}, r>0$ and $t \geq 1$. In this case we write $w \in D_{\mu}$. It is well known that if $w$ satisfies (i) or (ii) in $\S 1$ then $w \in D_{\mu}$ for some $\mu \geq 1$.

\section{The $L^{\infty}$-estimate}

In this section we will show the following

Theorem (3.1). Given $p>0$ there exist positive constants $r_{0}$ and $C$ only depending on $p, \lambda, n, \eta$ and $\Omega$ such that if $u$ is any solution of $L u-V u=0$ in $\Omega$ and $B_{r}$ is any ball with $r \leq r_{0}$ and $B_{2 r} \subset \Omega$ then we have

$$
\sup _{B_{r / 2}}|u| \leq C\left(f_{B_{r}}|u|^{p} w(x) d x\right)^{1 / p} .
$$

The proof will be a consequence of the following lemmas.

Lemma (3.2). Let $u$ be a solution of $L u-V u=0$ in $\Omega$. Then there exists $a$ positive constant $C=C\left(\lambda, n, c_{0}, \eta, \Omega\right)$ such that if $0<s<t$ and $B_{t} \subset \Omega$ then we have

$$
\int_{B_{s}}|\nabla u(x)|^{2} w(x) d x \leq C \frac{1}{(t-s)^{2}} \int_{B_{t}} u(x)^{2} w(x) d x .
$$

Proof. Take $\phi \in C_{0}^{\infty}\left(B_{t}\right)$ such that $0 \leq \phi \leq 1, \phi \equiv 1$ on $B_{s}$ and $\|\nabla \phi\|_{\infty} \leq$ $\frac{C}{t-s}$. We have

$$
\begin{aligned}
\int \mid \nabla & \left.u(x)\right|^{2} \phi(x)^{2} w(x) d x \leq \lambda \int\langle a(\nabla u), \nabla u\rangle \phi(x)^{2} d x \\
& =\lambda \int\left\langle a(\nabla u), \nabla\left(u \phi^{2}\right)\right\rangle d x-2 \lambda \int\langle a(\nabla u), \nabla \phi\rangle \phi(x) u(x) d x \\
& =-\lambda \int u(x)^{2} \phi(x)^{2} V(x) d x-2 \lambda \int\langle a(\nabla u), \nabla \phi\rangle \phi(x) u(x) d x .
\end{aligned}
$$

To estimate the second term we use the fact that for every $\varepsilon>0$ we have

$$
|\langle a(\phi \nabla u), u \nabla \phi\rangle| \leq \frac{\varepsilon}{2}\langle a(\phi \nabla u), \phi \nabla u\rangle+\frac{1}{2 \varepsilon}\langle a(u \nabla \phi), u \nabla \phi\rangle .
$$

Hence by taking $\varepsilon \lambda^{2}=1 / 2$ we obtain

$$
\int|\nabla u|^{2} \phi^{2} w d x \leq-2 \lambda \int u^{2} \phi^{2} V d x+4 \lambda^{4} \int u^{2}|\nabla \phi|^{2} w d x
$$

To estimate the second integral in the last inequality we use the following embedding lemma. (For a proof of this lemma in the unweighted case see [9, p. 138].) 
Lemma (3.3). Let $\Omega$ be an open, bounded and connected set in $R^{n}$ and let $V$ be a potential satisfying (1.2). Then given $\varepsilon>0$ there exists a constant $C_{\varepsilon}=C(\varepsilon, \Omega, w, n, V, \lambda)$ such that for any $u \in H_{0}^{1}(\Omega, w)$ we have

$$
\int u^{2}(x)|V(x)| d x \leq \varepsilon \int|\nabla u|^{2} w(x) d x+C_{\varepsilon} \int u^{2} w(x) d x .
$$

Proof. Given $D$ an open and bounded set in $R^{n}$ let $B_{R}$ be a ball such that $D \subset B_{R / 4}$ and let $G(x, y)$ be the Green's function for $L$ in $B_{R}$. We define

$$
\eta_{D}(s)=\sup _{x \in D} \int_{|x-y|<s}|V(y)| G(x, y) d y .
$$

By (1.3) we have that (1.2) is equivalent to

$$
\lim _{s \rightarrow 0} \eta_{D}(s)=0
$$

for every bounded and open set $D \subset R^{n}$. It is enough to prove the lemma for $u \in \operatorname{Lip}_{0}(\Omega)$, then the desired result follows by passing to the limit. Let us suppose first that $u$ has support contained in a ball $B_{r} \subset \Omega$, then we claim that for every $\varepsilon>0$ there exists a constant $C_{\varepsilon}$ independent of $u$ and $V$ such that

$$
\int u^{2} V d x \leq \varepsilon \int|\nabla u|^{2} w d x+C_{\varepsilon}\left[\eta_{B_{r}}(3 r) \int u^{2} V d x+\eta_{B_{r}}(3 r)^{2} \int|\nabla u|^{2} w d x\right] .
$$

To prove the claim assume $V$ has support contained in $B_{r}, V \geq 0$, and set

$$
F(x)=\int V(y) G(x, y) d y
$$

where $G$ is the Green's function of a ball $B$ such that $\frac{1}{4} B \supset \Omega$. Then $F$ is the solution of $L F=-V$ in $B$ and $F \in H_{0}^{1}(B, w)$. This is because since $V$ satisfies (1.2) then

$$
\int_{B} \int_{B} G(x, y) V(x) V(y) d x d y<\infty,
$$

and therefore as in the proof of Theorem 4.8 of [4] we can conclude that $\chi_{B} V \in$ $H^{-1}(B, w),\left(\chi_{B}\right.$ denotes the characteristic function of $\left.B\right)$. Therefore

$$
\begin{aligned}
\int u^{2} V d x & =\int\left\langle a(\nabla F), \nabla u^{2}\right\rangle d x=2 \int\langle a(u \nabla F), \nabla u\rangle d x \\
& \leq \varepsilon \int\langle a(\nabla u), \nabla u\rangle d x+\frac{1}{\varepsilon} \int\langle a(u \nabla F), u \nabla F\rangle d x \\
& \leq \lambda \varepsilon \int|\nabla u|^{2} w(x) d x+\frac{\lambda}{\varepsilon} \int|\nabla F|^{2} u^{2} w d x .
\end{aligned}
$$


Now observe that

$$
\begin{aligned}
\int|\nabla F|^{2} u^{2} w d x \leq \lambda \int\langle a(\nabla F), \nabla F\rangle u^{2} d x \\
\quad=\lambda \int\left\langle a(\nabla F), \nabla\left(F u^{2}\right)\right\rangle d x-2 \lambda \int\langle a(\nabla F), \nabla u\rangle u F d x \\
\quad=\lambda \int u^{2} F V d x-2 \lambda \int\langle a(u \nabla F), F \nabla u\rangle d x \\
\quad \leq \lambda \int u^{2} F(x) V(x) d x+\lambda \delta \int|\nabla F|^{2} u^{2} w d x+\frac{\lambda}{\delta} \int F^{2}|\nabla u|^{2} w d x
\end{aligned}
$$

for every $\delta>0$. Note that for $x \in B_{r}, F(x) \leq \eta_{B_{r}}(3 r)$ and then by taking $\delta=(2 \lambda)^{-1}$ we get

$$
\int|\nabla F|^{2} u^{2} w d x \leq 2 \eta_{B_{r}}(3 r) \lambda \int u^{2} V d x+4 \lambda^{2} \eta_{B_{r}}(3 r)^{2} \int|\nabla u|^{2} w d x .
$$

The claim follows with $C_{\varepsilon}=4 \lambda^{4} / \varepsilon$.

To complete the proof of the lemma, given $0<\delta<1$, let $\left\{\psi_{j}^{2}\right\}_{1}^{N}$ be a finite partition of unity of $\bar{\Omega}$ such that $\operatorname{supp} \psi_{j} \subseteq B_{r_{j}}\left(x_{j}\right)$ with $x_{j} \in \bar{\Omega}$ and $0<r_{j} \leq \delta$. Set $\tilde{\Omega}=\{x: d(x, \bar{\Omega}) \leq 1\}$. Therefore

$$
\begin{aligned}
\int\left(u \psi_{j}\right)^{2} V d x \leq & \frac{\varepsilon}{2}\left|\nabla\left(u \psi_{j}\right)\right|^{2} w d x \\
+ & C_{\varepsilon}\left[\eta_{B_{r_{j}}}\left(3 r_{j}\right) \int\left(\psi_{j} u\right)^{2} V d x+\eta_{B_{r_{j}}}\left(3 r_{j}\right)^{2} \int\left|\nabla\left(u \psi_{j}\right)\right|^{2} w d x\right] \\
\leq & \frac{\varepsilon}{2} \int|\nabla u|^{2} \psi_{j}^{2} w d x+\frac{\varepsilon}{2} \int u^{2}\left|\nabla \psi_{j}\right|^{2} w d x \\
+ & C_{\varepsilon}\left[\eta_{\tilde{\Omega}^{\prime}}(3 \delta) \int\left(\psi_{j} u\right)^{2} V d x\right. \\
& \left.+\eta_{\dot{\Omega}}(3 \delta)^{2} \int|\nabla u|^{2} \psi_{j}^{2} w d x+\eta_{\dot{\Omega}}(3 \delta)^{2} \int\left|\nabla \psi_{j}\right|^{2} u^{2} w d x\right] .
\end{aligned}
$$

We now choose $\delta=\delta(\varepsilon)<1$ such that

Hence

$$
C_{\varepsilon} \eta_{\tilde{\Omega}}(3 \delta)<\frac{1}{2} \quad \text { and } \quad C_{\varepsilon} \eta_{\tilde{\Omega}}(3 \delta)^{2}<\frac{\varepsilon}{2}
$$

$$
\frac{1}{2} \int\left(u \psi_{j}\right)^{2} V d x \leq \varepsilon \int|\nabla u|^{2} \psi_{j}^{2} w d x+\varepsilon \int u^{2}\left|\nabla \psi_{j}\right|^{2} w d x
$$

By summing in $j$ it follows that

$$
\frac{1}{2} \int u^{2} V d x \leq \varepsilon \int|\nabla u|^{2} w d x+\varepsilon \frac{N(\varepsilon)}{\delta(\varepsilon)^{2}} \int u^{2} w d x
$$

Remarks. (1) The constant $C_{\varepsilon}$ only depends on $\varepsilon, \Omega, \eta, n, c_{0}$ and $\lambda$.

(2) By Sobolev's inequality (2.3) Lemma (3.3) implies the following twoweights Sobolev inequality

$$
\int u^{2}(x) V(x) d x \leq C \int|\nabla u|^{2} w(x) d x
$$


Lemma (3.4). There exists a constant $C=C\left(n, \lambda, \eta, \Omega, c_{0}\right)$ such that if $u$ is a solution of $L u-V u=0$ in $\Omega$ and $B_{2 r}\left(x_{0}\right) \subset \Omega$ then

$$
\left(f_{B_{r / 2}\left(x_{0}\right)} u^{2} w d x\right)^{1 / 2} \leq C f_{B_{r}\left(x_{0}\right)}|u| w d x
$$

Proof. We claim that it is enough to prove the lemma when $r=1$ and $x_{0}=0$. In fact $u_{x_{0}}(x)=u\left(x-x_{0}\right)$ is defined in $\Omega+x_{0}$ and if $a_{x_{0}}(x)=$ $a\left(x-x_{0}\right), V_{x_{0}}(x)=V\left(x-x_{0}\right)$ and $w_{x_{0}}(x)=w\left(x-x_{0}\right)$ then $u_{x_{0}}$ is a solution of $\operatorname{div}\left(a_{x_{0}}(x) \nabla\right)-V_{x_{0}}=0$ in $\Omega+x_{0}$. Note that the constant $c_{0}$ of $w_{x_{0}}$ does not change and $V_{x_{0}}$ is in $K_{\eta}$ defined with $w_{x_{0}}$. Therefore by translations we can assume $x_{0}=0$. Set $u_{r}(x)=u(r x)$, then $u_{r}$ is defined in $\frac{1}{r} \Omega$ (in particular in $\left.B_{2}\right)$ and if we set $a_{r}(x)=a(r x), V_{r}(x)=r^{2} V(r x)$ and $w_{r}(x)=w(r x)$ then $u_{r}$ is a solution of $\operatorname{div}\left(a_{r}(x) \nabla\right)-V_{r}=0$ in $\frac{1}{r} \Omega$. Note again that the constant $c_{0}$ of $w_{r}$ does not change. Also by changing variables is easy to see that

$$
\begin{aligned}
& \sup _{x \in 1 / r \Omega} \int_{|x-y|<\delta}\left|V_{r}(y)\right| \int_{|x-y|}^{4 R / r} \frac{s}{w_{r}\left(B_{s}(x)\right)} d s d y \\
& \quad=\sup _{x \in \Omega} \int_{|x-y|<\delta r}|V(y)| \int_{|x-y|}^{4 R} \frac{s}{w\left(B_{s}(x)\right)} d s d y,
\end{aligned}
$$

which if $r \leq 1$ implies that $V_{r}$ belongs to the class $K_{\eta}$ defined with $w_{r}$. Let us assume $f_{B_{1}}|u| w d x=1$ and for $\frac{1}{2}<s<1$ consider

$$
I(s)=\left(\frac{1}{w\left(B_{1 / 2}\right)} \int_{B_{s}} u^{2} w d x\right)^{1 / 2} .
$$

If $I\left(\frac{1}{2}\right) \leq 1$ then there is nothing to show, so suppose $I\left(\frac{1}{2}\right)>1$. We want to show $I\left(\frac{1}{2}\right) \leq C, C$ only depends on $n, \lambda, \eta, \Omega$ and $c_{0}$. Let $\tau$ be the exponent in the Poincaré inequality and choose $0<\theta<1$ such that $(2-\theta) /(1-\theta)=2 \tau$. By doubling and Poincaré we have

$$
\begin{aligned}
I(s) & =\left(\frac{1}{w\left(B_{1 / 2}\right)} \int_{B_{s}}|u|^{2-\theta}|u|^{\theta} w d x\right)^{1 / 2} \leq C\left(f_{B_{s}}|u|^{(2-\theta) /(1-\theta)} w d x\right)^{(1-\theta) / 2} \\
& \leq C s^{(1-\theta) \tau}\left(f_{B_{s}}|\nabla u|^{2} w d x\right)^{((1-\theta) / 2) \tau}+C\left(f_{B_{s}}|u|^{2} w d x\right)^{((1-\theta) / 2) \tau} .
\end{aligned}
$$

If $\frac{1}{2} \leq s<t \leq 1$ then by Lemma (3.2) and doubling we obtain $I(s) \leq$ $C\left[(t-s)^{-1} I(t)\right]^{(1-\theta) \tau}$ which implies $I\left(\frac{1}{2}\right) \leq C$. (See Lemma 1.2 of [1].)

Lemma (3.5). Let $\Omega=B_{2}(0), w \in D_{\mu}$ and $p>(n / 2) \mu$. There exist constants $\delta_{0}=\delta_{0}\left(\lambda, n, \eta, c_{0}\right)$ and $C=C\left(\lambda, n, \eta, c_{0}\right)$ such that if

$$
\sup _{B_{2}} \int_{B_{6}}|V(y)| \int_{|x-y|}^{8} \frac{s}{w\left(B_{s}(x)\right)} d s d y<\delta_{0}
$$


then given $f / w \in L_{w}^{p}(\Omega)$ there exists a unique $u \in H_{0}^{1}(\Omega, w)$ such that $L u-$ $V u=f$ in $\Omega$ and

Proof. The bilinear form

$$
\|u\|_{L^{\infty}(\Omega)} \leq \frac{c}{w\left(B_{1}\right)^{1 / p}}\|f / w\|_{L_{w}^{p}(\Omega)} .
$$

$$
\alpha(u, v)=\int_{\Omega}\langle a(\nabla u), \nabla v\rangle d x+\int_{\Omega} u v V d x
$$

is continuous and coercive in $H_{0}^{1}(\Omega, w)$ provided $\delta_{0}$ is small enough. This follows by the claim made in the proof of Lemma (3.3). Now if $p \geq 2$ and $f \in L_{w^{1-p}}^{p}(\Omega)$ implies $f \in H^{-1}(\Omega, w)$ and consequently the existence and uniqueness of $u$ is a consequence of the Lax-Milgram theorem, (see [7]).

Let $u_{0}$ be the solution of the problem $L u=f$ in $\Omega, u / \partial \Omega=0$ (i.e. $\left.u \in H_{0}^{1}(\Omega, w)\right)$, and for $j \geq 1$, let $u_{j}$ be the solution of $L u-V u_{j-1}=f$ in $\Omega, u / \partial \Omega=0$. Then we have

$$
u_{0}(x)=\int_{\Omega} G_{L}(x, y) f(y) d y,
$$

where $G_{L}(x, y)$ is the Green's function of $L$ in $\Omega$. By the maximum principle

$$
G_{L}(x, y) \leq C \int_{|x-y|}^{8} \frac{s}{w\left(B_{s}(x)\right)} d s .
$$

It is easy to see that

$$
\left(\int_{\Omega} G_{L}(x, y)^{p^{\prime}} w(y) d y\right)^{1 / p^{\prime}}=\frac{C}{w\left(B_{1}(0)\right)^{1 / p}}=C_{1}<\infty, \quad \text { for } p>\frac{n}{2} \mu .
$$

Then

We also have

$$
\left|u_{0}(x)\right| \leq C_{1}\|f / w\|_{L_{w}^{p}(\Omega)}, \quad x \in \Omega
$$

$$
u_{1}(x)=\int_{\Omega} G_{L}(x, y) f(y) d y+\int_{\Omega} G_{L}(x, y) V(y) u_{0}(y) d y
$$

which implies

$$
\left|u_{1}(x)\right| \leq C_{1}\|f\|_{L_{w^{1}-p}^{p}}+C_{1} \delta\|f\|_{L_{w^{1}-p}^{p}}
$$

provided $\sup _{\Omega} \int G_{L}(x, y)|V(y)| d y<\delta$. Continuing in this manner we obtain

$$
u_{j}(x)=\int_{\Omega} G_{L}(x, y) f(y) d y+\int_{\Omega} G_{L}(x, y) V(y) u_{j-1}(y) d y
$$

and

$$
\left|u_{j}(x)\right| \leq C_{1} \cdot C_{\delta}\|f / w\|_{L_{w}^{p}(\Omega)}, \quad \text { for } j=2,3, \ldots
$$

We claim that $u_{j}$ is a Cauchy sequence in $H_{0}^{1}(\Omega, w)$ and $u_{j} \rightarrow u$ in $H_{0}^{1}(\Omega, w)$. By (3.6) we have

$$
u_{j+1}(x)-u_{j}(x)=\int_{\Omega} G_{L}(x, y) V(y)\left[u_{j}(y)-u_{j-1}(y)\right] d y
$$


and therefore

$$
\left\|u_{j+1}-u_{j}\right\|_{L_{(\Omega)}^{\infty}} \leq \delta\left\|u_{j}-u_{j-1}\right\|_{L_{(\Omega)}}
$$

Consequently for $m>n$

$$
\begin{aligned}
\left\|u_{m}-u_{n}\right\|_{L^{\infty}(\Omega)} & \leq \sum_{j=n}^{m-1}\left\|u_{j+1}-u_{j}\right\|_{L^{\infty}(\Omega)} \\
& \leq\left\|u_{0}-u_{1}\right\|_{L^{\infty}(\Omega)} \sum_{j=n}^{m-1} \delta^{j}
\end{aligned}
$$

Therefore for $\delta<1 \quad\left\{u_{j}\right\}$ is a Cauchy sequence in $L^{\infty}(\Omega)$ and therefore in $L^{2}(\Omega, w)$. Also

$$
\begin{aligned}
\int_{\Omega} \mid & \left.\nabla\left(u_{m}-u_{n}\right)\right|^{2} w(x) d x \leq \lambda \int_{\Omega}\left\langle a\left(\nabla\left(u_{m}-u_{n}\right)\right), \nabla\left(u_{m}-u_{n}\right)\right\rangle d x \\
& =-\int\left(u_{m}-u_{n}\right)\left(u_{m-1}-u_{n-1}\right) V(x) d x \\
& \leq\left(\int\left|u_{m}-u_{n}\right|^{2} V(x) d x\right)^{1 / 2}\left(\int\left|u_{m-1}-u_{n-1}\right|^{2} V(x) d x\right)^{1 / 2},
\end{aligned}
$$

and since $V \in L^{1}(\Omega)$ we have $\left\|\nabla\left(u_{m}-u_{n}\right)\right\|_{L^{2}(\Omega, w)}$ tends to 0 as $m, n \rightarrow \infty$. Consequently $u_{j} \rightarrow \tilde{u}$ in $H_{0}^{1}(\Omega, w)$ and by (3.6) $\tilde{u}=u$.

Remark (3.7). Lemma (3.5) implies the existence and integrability of the Green's function $G(x, y)$ of $L-V$. In fact, if $p>(n / 2) \mu$ then

$$
\int_{\Omega} G(x, y) w(y)^{(p-1) / p} f(y) w(y)^{(1-p) / p} d y \leq \frac{C}{w\left(B_{1}\right)^{1-p}}\left\|f w^{(1-p) / p}\right\|_{L^{p}(\Omega)},
$$

which implies

$$
\left(\int_{\Omega} G(x, y)^{q} w(y) d y\right)^{1 / q}=\frac{C}{w\left(B_{1}\right)^{1 / p}}<\infty \text { for } 1<q<\frac{n \mu}{n \mu-2}
$$

and a.e. $x \in \Omega\left(\frac{1}{p}+\frac{1}{9}=1\right)$.

Theorem 3.8. There exist $r_{0}=r_{0}\left(\lambda, n, c_{0}, \eta\right)$ and for each $p>0$ a constant $C=C\left(p, \lambda, n, c_{0}, \eta\right)$ such that if $u$ is a solution of $L u-V u=0$ in $\Omega$ and $B_{2 r} \subset \Omega$ with $r \leq r_{0}$ then

$$
\sup _{B_{r / 2}}|u| \leq C\left(f_{B_{r}}|u(x)|^{p} w(x) d x\right)^{1 / p} .
$$

Proof. By translation we may assume $B_{r}$ is centered at 0 . As before $u_{r}(x)=$ $u(r x)$ is a solution in $B_{2}=B_{2}(0)$ of $L_{r} u_{r}-V_{r} u_{r}=0$ where $L_{r}$ and $V_{r}$ are defined in Lemma (3.4). Also observe that

$$
\begin{aligned}
\int_{|x-y|<\delta} & \left|V_{r}(y)\right| \int_{|x-y|}^{8} \frac{s}{w_{r}\left(B_{s}(x)\right)} d s d y \\
& =\int_{|r x-z|<r \delta} \int_{|r x-z|}^{8 r} \frac{s}{w_{r}\left(B_{s}(x)\right)} d s d y \leq \eta(\delta r) .
\end{aligned}
$$


Therefore

$$
\sup _{x \in B_{2}} \int_{|x-y|<\delta}\left|V_{r}(y)\right| \int_{|x-y|}^{8} \frac{s}{w_{r}\left(B_{s}(x)\right)} d s d y \leq \eta(\delta r) .
$$

Since $\eta(\delta r) \rightarrow 0$ as $\delta \rightarrow 0$ then it is enough to show that if $\delta_{0}$ is the number specified in Lemma (3.5) and

$$
\sup _{B_{2}} \int_{B_{6}}|V(y)| \int_{|x-y|}^{8} \frac{s}{w\left(B_{s}(x)\right)} d s d y<\delta_{0}
$$

then we have

$$
\sup _{B_{1 / 2}}|u| \leq C\left(f_{B_{1}}|u|^{p} w(y) d y\right)^{1 / p},
$$

with $C=C\left(\lambda, \eta, p, n, c_{0}\right)$.

Let $G(x, y)$ be the Green's function of $L-V$ in $B_{2}$. Given $\frac{1}{2} \leq s<t \leq 1$, let $\psi$ be in $C_{0}^{\infty}\left(B_{t-(t-s) / 4}(0)\right)$ such that $0 \leq \psi \leq 1, \psi \equiv 1$ on $B_{(t+s) / 2}(0)$ and $|\nabla \psi| \leq C /(t-s)$. We have

$$
\begin{gathered}
u(x) \psi(x)=\int_{B_{2}}\left\langle a\left(\nabla_{y} G(x, y)\right), \nabla \psi(y)\right\rangle u(y) d y \\
-\int_{B_{2}}\langle a(\nabla u), \nabla \psi\rangle G(x, y) d y=J_{1}-J_{2} . \\
J_{1}=\int_{B_{t-(t-s) / 4} \backslash B_{(t+s) / 2}}\langle a(\nabla G(x, \cdot)), \nabla \psi\rangle u(y) d y \\
\leq\left(\int_{B_{t-(t-s) / 4} \backslash B_{(t+s) / 2}}\left|\nabla_{y} G(x, y)\right|^{2} w(y) d y\right)^{1 / 2} \\
\quad \times\left(\int_{B_{t-(t-s) / 4} \backslash B_{(t+s) / 2}} u^{2}|\nabla \psi|^{2} w(y) d y\right)^{1 / 2} \\
\leq \frac{C}{t-s}\left(\int_{B_{t-(t-s) / 4} \backslash B_{(t+s) / 2}}\left|\nabla \nabla_{y} G(x, y)\right|^{2} w(y) d y\right)^{1 / 2}\left(\int_{B_{t}} u^{2} w(y) d y\right)^{1 / 2} .
\end{gathered}
$$

Analogously

Now by Lemma (3.2)

$$
\begin{aligned}
J_{2} \leq & \frac{C}{t-s}\left(\int_{B_{t-(t-s) / 4} \backslash B_{(t+s) / 2}}|G(x, y)|^{2} w(y) d y\right)^{1 / 2} \\
& \times\left(\int_{B_{t-(1-s) / 4} \backslash B_{(t+s) / 2}}|\nabla u|^{2} w(y) d y\right)^{1 / 2} .
\end{aligned}
$$

$$
\begin{aligned}
& \int_{B_{t-(1-s) / 4} \backslash B_{t(t+s) / 2}}|\nabla u|^{2} w(y) d y \leq \int_{B_{t-(t-s) / 4}}|\nabla u|^{2} w(y) d y \\
& \quad \leq \frac{C}{(t-s)^{2}} \int_{B_{l}} u^{2} w(y) d y .
\end{aligned}
$$


We cover the annulus $B_{t-(t-s) / 4} \backslash B_{(t+s) / 2}$ by a union of $N$ balls $B_{(t-s) / 4}\left(z_{i}\right)$, with $\left|z_{i}\right|=(t+s) / 2+(t-s) / 8$ (observe that the annulus has width $(t-s) / 4$ ). Therefore if $x \in B_{s}$ then $x \notin B_{(t-s) / 2}\left(z_{i}\right)$ and then for $x \in B_{s}$ we have

$$
\begin{gathered}
\int_{B_{t-(t-s) / 4} \backslash B_{(t+s) / 2}}\left|\nabla_{y} G(x, y)\right|^{2} w(y) d y \leq \sum_{i=1}^{N} \int_{B_{(t-s) / 4}\left(z_{i}\right)}|\nabla G(x, y)|^{2} w(y) d y \\
\leq \sum_{i=1}^{N} \frac{C}{(t-s)^{2}} \int_{B_{(t-s) / 2}\left(z_{i}\right)}|G(x, y)|^{2} \\
\quad=\frac{C}{(t-s)^{2}} \sum_{i=1}^{N} w\left(B_{(t-s) / 2}\left(z_{i}\right)\right) f_{B_{(t-s) / 2}\left(z_{i}\right)}|G(x, y)|^{2} w(y) d y
\end{gathered}
$$

which by Lemma (3.4) is less than

$$
\frac{C}{(t-s)^{2}} \sum_{i=1}^{N} w\left(B_{t-s}\left(z_{i}\right)\right)^{-1} \cdot\left(\int_{B_{t-s}\left(z_{i}\right)} G(x, y) w(y) d y\right)^{2} .
$$

Now by doubling we have $w\left(B_{1}(0)\right)(t-s)^{n \mu} \leq c \cdot w\left(B_{t-s}\left(z_{i}\right)\right)$ and then by Remark (3.7) we obtain

$$
\left(\int_{B_{t-(1-s) / 4} \backslash B_{(t+s) / 2}}|\nabla G(x, y)|^{2} w(y) d y\right)^{1 / 2} \leq \frac{C}{(t-s)^{1+(n / 2) \mu}} \frac{1}{w\left(B_{1}\right)^{1 / 2}} .
$$

Analogously we have

$$
\begin{aligned}
& \int_{B_{t-(t-s) / 4} \backslash B_{(t+s) / 2}} G(x, y)^{2} w(y) d y \\
& \leq C \sum_{i=1}^{N} w\left(B_{t-s}\left(z_{i}\right)\right)\left(f_{B_{(t-s) / 2}\left(z_{i}\right)} G(x, y) w(y) d y\right)^{2} .
\end{aligned}
$$

Collecting estimates we obtain

$$
\|u\|_{L^{\infty}{ }_{\left(B_{s}\right)}} \leq \frac{C}{(t-s)^{2+(n / 2) \mu}}\left(\frac{1}{w\left(B_{1}\right)} \int_{B_{t}} u^{2} w(y) d y\right)^{1 / 2} .
$$

For $\frac{1}{2} \leq s \leq 1$ we set $I(s)=\left(1 / w\left(B_{1}\right) \int_{B_{s}} u^{2} w d x\right)^{1 / 2}$. Let $p>0$ and assume $f_{B_{1}} u^{p} w d y=1$, then if $p<2$ we have

$$
I(s) \leq\left(\sup _{B_{s}}|u|\right)^{\theta}, \quad \theta=1-\frac{p}{2},
$$

and therefore

$$
I(s) \leq \frac{C}{(t-s)^{(2+(n / 2) \mu) \theta}} I(t)^{\theta} .
$$

By the argument in $[6$, p. 1004] we obtain the theorem. 
414

C. E. GUTIERREZ

4. The INFIMUM estimate

We begin with the following

Lemma (4.1). Let $u \geq 0$ be a solution of $L u-V u=0$ in $\Omega$. Then there exists a constant $C=C\left(\lambda, \eta, c_{0}\right)$ independent of $u$ such that if $B_{4 r} \subset \Omega$ then for every $\varepsilon>0$ we have

$$
\frac{1}{w\left(B_{r}\right)} \int_{B_{r}}\left|\log (u+\varepsilon)-f_{B_{r}} \log (u+\varepsilon) w(y) d y\right|^{2} w(y) d y \leq C .
$$

Proof. Let $\psi \in C_{0}^{\infty}\left(B_{3 r / 2}\right), \psi \equiv 1$ on $B_{r}$ and $0 \leq \psi \leq 1,|\nabla \psi| \leq c / r$, $B_{r}=B_{r}(x)$ and set $u_{\varepsilon}=u+\varepsilon$. Then

$$
\begin{aligned}
\int\left|\nabla \log u_{\varepsilon}\right|^{2} w d y & =\int \frac{\left|\nabla u_{\varepsilon}\right|^{2}}{u_{\varepsilon}^{2}} \psi^{2} w d y \\
& \leq \lambda \int\left\langle a\left(\nabla u_{\varepsilon}\right), \nabla u_{\varepsilon}\right\rangle \frac{\psi^{2}}{u_{\varepsilon}^{2}} d y \\
& =2 \lambda \int\langle a(\nabla u), \nabla \psi\rangle \frac{\psi}{u_{\varepsilon}} d y-\lambda \int\left\langle a(\nabla u), \nabla\left(\frac{\psi^{2}}{u_{\varepsilon}}\right)\right\rangle d y \\
& =2 \lambda \int\langle a(\nabla u), \nabla \psi\rangle \frac{\psi}{u_{\varepsilon}} d y+\lambda \int V(y) \frac{u}{u_{\varepsilon}} \psi^{2} d y \\
& \leq 2 \lambda \int\langle a(\nabla u), \nabla \psi\rangle \frac{\psi}{u_{\varepsilon}} d y+\lambda \int|V(y)| \psi^{2} d y .
\end{aligned}
$$

We have

$$
\begin{aligned}
& \int V(y) \psi^{2}(y) d y \\
& \quad \leq \int_{B_{2 r}(x)}|V(y)|\left(\int_{|x-y|}^{4 r} \frac{s}{w\left(B_{s}(x)\right)} d s\right)\left(\int_{|x-y|}^{4 r} \frac{s}{w\left(B_{s}(x)\right)} d s\right)^{-1} d y .
\end{aligned}
$$

If $y \in B_{2 r}$ then by doubling we have

$$
\int_{|x-y|}^{4} \frac{s}{w\left(B_{s}(x)\right)} d s \geq C \cdot \frac{r^{2}}{w\left(B_{r}(x)\right)} .
$$

Consequently by the assumption on $V$ we have

$$
\int V(y) \psi^{2}(y) d y \leq C \frac{w\left(B_{r}(x)\right)}{r^{2}} .
$$

Also as in the proof of Lemma (3.2) we have for every $\delta>0$

$$
\int\langle a(\nabla u), \nabla \psi\rangle \frac{\psi}{u_{\varepsilon}} d y \leq \frac{\lambda}{\delta} \int\left|\nabla \log u_{\varepsilon}\right|^{2} \psi^{2} w(y) d y+\delta \cdot C \frac{w\left(B_{r}(x)\right)}{r^{2}} .
$$

Hence if $\delta$ is large we have

$$
\int\left|\nabla \log u_{\varepsilon}\right|^{2} w d y \leq C \frac{w\left(B_{r}(x)\right)}{r^{2}} .
$$


Now since $\log u_{\varepsilon} \in H^{1}\left(B_{2 r}, w\right)$ then by Poincare the lemma follows.

Remark (4.2). Note that if $w \in A_{\infty}$ then by Theorem 5 of [8], Lemma (4.1) implies that $\log (u+\varepsilon) \in B M O, \varepsilon>0$.

Lemma (4.3). Let $u>0$ be a solution of $L u-V u=0$ in $\Omega$ and let $r_{0}$ be the number in Theorem (3.8) then there exists a constant $C=C\left(\lambda, \eta, n, c_{0}\right)$ such that

$$
\int_{B_{2 r}} u(x) w(x) d x \leq C \int_{B_{r}} u(x) w(x) d x
$$

for $0<r \leq r_{0}$ and $B_{8 r} \subset \Omega$.

Proof. By Lemma (4.1) and Theorem 5 of [8] there exist $\delta>0$ and $C>0$ such that

$$
\left(\frac{1}{w\left(B_{r}\right)} \int_{B_{r}} u_{\varepsilon}^{\delta} w d x\right)\left(\frac{1}{w\left(B_{r}\right)} \int_{B_{r}} u_{\varepsilon}^{-\delta} w d x\right) \leq C,
$$

for $B_{4 r} \subset \Omega$, i.e. $u_{\varepsilon}^{\delta} \in A_{2}(w)$, for every $\varepsilon>0$. Since $w$ is doubling this implies

$$
\int_{B_{2 r}} u_{\varepsilon}^{\delta} w d x \leq C \int_{B_{r}} u_{\varepsilon}^{\delta} w d x
$$

Hence by Theorem (3.8) and $\delta<1$ we have

$$
\begin{aligned}
\frac{1}{w\left(B_{2 r}\right)} \int_{B_{2 r}} u w d x & \leq C\left(\frac{1}{w\left(B_{4 r}\right)} \int_{B_{4 r}} u_{\varepsilon}^{\delta} w d x\right)^{1 / \delta} \\
& \leq C\left(\frac{1}{w\left(B_{r / 2}\right)} \int_{B_{r / 2}} u_{\varepsilon}^{\delta} w d x\right)^{1 / \delta} \leq \frac{1}{w\left(B_{r}\right)} \int_{B_{r}} u_{\varepsilon} w d x,
\end{aligned}
$$

by letting $\varepsilon \rightarrow 0$ this implies the lemma.

Lemma (4.4). Set $V^{+}=\max (V, 0)$ and let $G(x, y)$ denote the Green's function of $L-V^{+}$in $\Omega$. Then for any $1<q<n \mu /(n \mu-2) \quad\left(w \in D_{\mu}\right)$ there exists a constant $C=C\left(q, \lambda, \eta, c_{0}\right)$ such that

$$
\left(\frac{1}{w\left(B_{r}\right)} \int_{B_{r}} G(x, y)^{q} w(y) d y\right)^{1 / q} \leq C \frac{1}{w\left(B_{r}\right)} \int_{B_{r}} G(x, y) w(y) d y,
$$

for $B_{8 r} \subset \Omega$ and $0<r \leq r_{0} \quad\left(r_{0}\right.$ is as in Theorem (3.8)).

Proof. First observe that $G(x, \cdot)$ is a solution of $L-V^{+}=0$ for $y \neq x$, $x \in \Omega$. Suppose first that $x \notin B_{8 r}$, then by Theorem (3.8) we have for every $q>0$ that

$$
\left(f_{B_{r}} G(x, y)^{q} w(y) d y\right)^{1 / q} \leq \sup _{y \in B_{r}} G(x, y) \leq C f_{B_{2 r}} G(x, y) w(y) d y
$$

which by Lemma (4.3) is less than

$$
f_{B_{r}} G(x, y) w(y) d y .
$$


Now assume $x \in B_{8 r}$ and let $G_{r}(x, y)=G_{L-V^{+}, B_{8 r}}(x, y)$. By the maximum principle $G_{r}(x, y) \leq G(x, y)$ for $x, y \in B_{8 r}$. Then

$$
\begin{aligned}
f_{B_{r}} G(x, y)^{q} w(y) d y \leq 2^{q}\left\{f_{B_{r}}\left[G(x, y)-G_{r}(x, y)\right]^{q} w(y) d y\right. & \\
& \left.+f_{B_{r}} G_{r}(x, y)^{q} w(y) d y\right\} .
\end{aligned}
$$

Since $G(x, \cdot)-G_{r}(x, \cdot)$ is a nonnegative solution of $L-V^{+}=0$ in $B_{8 r}$ then arguing as before we obtain

$$
f_{B_{r}}\left[G(x, y)-G_{r}(x, y)\right]^{q} w(y) d y<C\left\{f_{B_{r}}\left[G(x, y)-G_{r}(x, y)\right] w(y) d y\right\}^{q}
$$

By translation we can assume that $B_{r}$ is centered at 0 . Let $L_{r}$ and $V_{r}^{+}$be defined as in Lemma (3.4) and let $\tilde{G}(x, y)$ be the Green's function to $L_{r}-V_{r}^{+}$ in $B_{8}$. Then for $x \in B_{8 r}$ and $z \in B_{8}$ we have

$$
G_{r}(x, r z)=r^{2-n} \tilde{G}(x, z) .
$$

Therefore

$$
f_{B_{r}} G_{r}(x, y)^{q} w(y) d y=\frac{r^{n}}{w\left(B_{r}\right)}\left(\int_{|z| \leq 1} \tilde{G}(x, z)^{q} w(r z) d z\right) r^{(2-n) q}
$$

and

$$
f_{B_{r}} G_{r}(x, y) w(y) d y=\frac{r^{n}}{w\left(B_{r}\right)}\left(\int_{B_{1}} \tilde{G}(x, z) w(r z) d z\right) r^{2-n}
$$

Then if we set

$$
u(x)=\int_{B_{1}} \tilde{G}(x, z) w(r z) d z
$$

then $\left(L_{r}-V_{r}^{+}\right) u=-\chi_{B_{1}} w_{r}\left(w_{r}(z)=w(r z)\right)$. Since $u / \partial B_{8}=0$ then we have

$$
\begin{aligned}
u(x)= & \int_{B_{1}} G_{L_{r}, B_{8}}(x, y) w_{r}(y) d y \\
& -\int_{B_{1}} G_{L_{r}, B_{8}}(x, y) V_{r}^{+}(y) u(y) d y
\end{aligned}
$$


where $G_{L_{r}, B_{8}}$ is the Green's function to $L_{r}$ in $B_{8}$. By the estimates (1.3) for $G_{L_{r}, B_{8}}$ we have

$$
\begin{aligned}
\int_{B_{1}} G_{L_{r}, B_{8}}(x, y) w_{r}(y) d y & \geq C \int_{B_{1}}\left(\int_{|x-y|}^{8} \frac{s}{w_{r}\left(B_{s}(x)\right)} d s\right) w_{r}(y) d y \\
& =C \int_{B_{1}} \int_{0}^{8} \chi_{(|x-y|, 8)}(s) \frac{s}{w_{r}\left(B_{s}(x)\right)} d s w_{r}(y) d y \\
& =C \int_{0}^{8} \frac{s}{w_{r}\left(B_{s}(x)\right)} \int_{B_{1}} \chi_{(|x-y|, 8)}(s) w_{r}(y) d y d s \\
& =C \int_{0}^{8} \frac{s}{w_{r}\left(B_{s}(x)\right)} w_{r}\left(B_{1} \cap B_{s}(x)\right) d s \\
& \geq C \int_{4}^{8} \frac{s}{w_{r}\left(B_{s}(x)\right)} w_{r}\left(B_{1} \cap B_{s}(x)\right) d s .
\end{aligned}
$$

If $x \in B_{3}$ and $s>4$ then $B_{1} \subset B_{s}(x)$ and by doubling we have

$$
\inf _{x \in B_{3}} \int_{B_{1}} G_{L_{r}, B_{8}}(x, y) w_{r}(y) d y \geq C
$$

$C$ independent of $r$. Also by (1.2) we have

$$
\int_{B_{1}} G_{L_{r}, B_{8}}(x, y) V^{+}(y) u(y) d y \leq \delta\|u\|_{L} \infty_{\left(B_{1}\right)} .
$$

Now by Lemma (3.5) (with $f=\chi_{B_{1}} w_{r}$ ) we have $\|u\|_{L} \infty_{\left(B_{1}\right)} \leq C$ with $C=$ $C\left(\lambda, n, \eta, c_{0}\right)$. This implies that there exist $\delta, C>0$ depending only on the parameters such that if

$$
\sup _{B_{4}} \int_{B_{4}}\left|V_{r}(y)\right| \int_{|x-y|}^{8} \frac{s}{w\left(B_{s}(x)\right)} d s d y \leq \delta
$$

then we have

$$
\inf _{B_{3}} u \geq C_{1} \text {. }
$$

Consequently for $1<q<n \mu /(n \mu-2)$ we have

$$
\begin{aligned}
f_{B_{r}} G_{r}(x, y)^{q} w(y) d y= & \frac{r^{n}}{w\left(B_{r}\right)}\left(\int_{|z| \leq 1} \tilde{G}(x, z)^{q} w_{r}(z) d z\right) r^{(2-n) q} \frac{u(x)^{q}}{u(x)^{q}} \\
\leq & C\left[\frac{r^{n}}{w\left(B_{r}\right)}\right]^{1-q}\left(f_{B_{r}} G_{r}(x, y) w(y) d y\right)^{q} \\
& \times\left(\int_{|z| \leq 1} \tilde{G}(x, z)^{q} w_{r}(z) d z\right) .
\end{aligned}
$$

By Remark (3.7) we have

$$
\int_{|z| \leq 1} \tilde{G}(x, z)^{q} w_{r}(z) d z \leq \frac{c}{w_{r}\left(B_{1}\right)^{q-1}},
$$

and since $w_{r}\left(B_{1}\right)=r^{-n} w\left(B_{r}\right)$ then the lemma follows. 
We are now in a position to show

Theorem (4.5). Let $u$ be a nonnegative solution of $L u-V u=0$ in $\Omega$. There exist positive constants $r_{0}=r_{0}\left(\lambda, \eta, n, c_{0}\right), p_{0}=p_{0}\left(\lambda, c_{0}\right)$ and $C=$ $C\left(\lambda, \eta, n, c_{0}\right)$ all independent of $u$ such that

$$
\left(f_{B_{r}} u^{p_{0}} w(y) d y\right)^{1 / p_{0}} \leq C \inf _{B_{r / 2}} u
$$

for $B_{8 r} \subset \Omega$ and $r \leq r_{0}$.

Proof. By translation and dilation we can assume $\Omega=B_{2}(0)$ and as in the proof of Theorem (3.8) we can assume $r=1$ and all balls are centered at 0 . We show that if $u \geq 1$ on a closed set $\Gamma \subset B_{1}$ in the sense of $H^{1}\left(B_{2}\right)$ then we have

$$
\inf _{B_{1 / 2}} u \geq C\left[\frac{w(\Gamma)}{w\left(B_{1}\right)}\right]^{M},
$$

where $C$ and $M$ only depend on $\lambda, \eta$ and $c_{0}$. Set

$$
z(x)=\int_{\Gamma} G_{L-V^{+}, B_{2}}(x, y) w(y) d y,
$$

then $\left(L-V^{+}\right) z=-\chi_{\Gamma} w$ in $B_{2}$. Also by Remark (3.7) we have

$$
z(x) \leq\left(\int_{B_{2}} G_{L-V^{+}, B_{2}}(x, y)^{q} w(y) d y\right)^{1 / q} w(\Gamma)^{1 / q^{\prime}} \leq C_{1}, \quad \text { a.e. in } B_{2},
$$

here $C_{1}$ only depends on $\lambda, n, \eta$ and $c_{0}$. Consequently $\left(1 / C_{1}\right) z(x) \leq 1$ in the $H^{1}$ sense in $B_{2}$ and therefore $\left(1 / C_{1}\right) z(x) \leq 1$ in the $H^{1}$ sense in $\Gamma$. Then $u(x) \geq\left(1 / C_{1}\right) z(x)$ in $H^{1}$ sense in $\Gamma$. Since $z / \partial B_{2}=0$ and $u \geq$ 0 in $B_{2}$ a.e. then we have $u \geq\left(1 / C_{1}\right) z$ in the $H^{1}$ sense in $\partial B_{2}$. Also $\left(L-V^{+}\right)\left(u-\left(1 / C_{1}\right) z\right)=-V^{-} u-\left(1 / C_{1}\right) \chi_{\Gamma} w \leq 0$, in $B_{2} \backslash \Gamma$, then by the maximum principle we have $u(x) \geq\left(1 / C_{1}\right) z(x)$ in $H^{1}$ sense in $B_{2}$ and consequently a.e. in $B_{2}$. Now Lemma (4.4) implies that there exists $C>0$ and $M>0$ such that

$$
\int_{\Gamma} G_{L-V^{+}, B_{2}}(x, y) w(y) d y \geq C\left[\frac{w(\Gamma)}{w\left(B_{1}\right)}\right]^{M} \int_{B_{1 / 4}} G_{L-V^{+}, B_{2}}(x, y) w(y) d y .
$$

By the argument used to prove Lemma (4.4) and (1.2) we obtain

$$
\inf _{x \in B_{1 / 2}} \int_{B_{1 / 4}} G_{L-V^{+}, B_{2}}(x, y) w(y) d y \geq C,
$$

where $C=C\left(\lambda, n, \eta, c_{0}\right)$. This implies (4.6). We claim that

$$
\inf _{B_{1 / 4}} u \geq C\left[\frac{w\left\{x \in B_{1}: u(x) \geq 2 \text { a.e. }\right\}}{w\left(B_{1}\right)}\right]^{M} \text {. }
$$

In fact, since $u \in H^{1}\left(B_{3 / 2}\right)$ there is a sequence $u_{n} \in \operatorname{Lip}\left(B_{3 / 2}\right)$ such that $u_{n} \rightarrow u$ in the $H^{1}\left(B_{3 / 2}\right)$ sense and a.e. Let $F=\left\{x \in B_{1}: u(x) \geq 2\right.$ a.e. $\}$, 
then by Egorov's theorem given $\varepsilon>0$ there exist a closed set $F_{\varepsilon} \subset F$ such that $w\left(F-F_{\varepsilon}\right)<\varepsilon$ and $u_{n} \rightarrow u$ uniformly in $F_{\varepsilon}$. Therefore $u \geq 1$ in the $H^{1}\left(B_{3 / 2}\right)$ sense in $F_{\varepsilon}$ and then

$$
\inf _{B_{1 / 2}} u \geq C\left[\frac{w\left(\Gamma_{\varepsilon}\right)}{w\left(B_{1}\right)}\right]^{M} \geq C\left[\frac{w(F)-\varepsilon}{w\left(B_{1}\right)}\right]^{M} .
$$

Now letting $\varepsilon \rightarrow 0$ the claim follows. By taking $u / t$ we obtain that

$$
\inf _{B_{1 / 2}} u \geq C t\left[\frac{w\left\{x \in B_{1}: u(x) \geq 2 t \text { a.e. }\right\}}{w\left(B_{1}\right)}\right]^{M},
$$

and consequently the theorem follows for $0<p_{0}<1 / M$.

\section{REFERENCES}

1. F. Chiarenza, E. Fabes and N. Garofalo, Harnack's inequality for Schrödinger operators and the continuity of solutions, Proc. Amer. Math. Soc. 98 (1986), 415-425.

2. R. Coifman and C. Fefferman, Weighted norm inequalities for maximal functions and singular integrals, Studia Math. 51 (1974), 241-250.

3. G. Dal Maso and U. Mosco, Wiener criteria and energy decay for relaxed Dirichlet problems, Arch. Rational Mech. Anal. 95 (1986), 347-387.

4. E. Fabes. D. Jerison and C. Kenig, The Wiener test for degenerate elliptic equations, Ann. Inst. Fourier (Grenoble) 32 (1982), 151-182.

5. E. Fabes, C. Kenig and R. Serapioni, The local regularity of solutions of degenerate elliptic equations, Comm. Partial Differential Equations 7 (1982), 77-116.

6. E. Fabes and D. Stroock, The $L^{p}$-integrability of Green's functions and fundamental solutions for elliptic and parabolic equations, Duke Math. J. 51 (1984), 997-1016.

7. D. Kinderlehrer and G. Stamppachia, An introduction to variational inequalities and their applications, Academic Press, New York, 1980.

8. B. Muckenhoupt and R. Wheeden, Weighted bounded mean oscillation and the Hilbert transform, Studia Math. 54 (1976), 221-237.

9. M. Schechter, Spectra of partial differential operators, North-Holland, Amsterdam, 1971.

Department of Mathematics, Temple University, Philadelphia, Pennsylvania 19122 